\title{
Disrupted Cerebellar Connectivity With the Central Executive Network and the Default-Mode Network in Unmedicated Bipolar II Disorder
}

\author{
Xiaomei Luo ${ }^{1,2 t}$, Guanmao Chen ${ }^{1,2 t}$, Yanbin Jia ${ }^{3}$, JiaYing Gong ${ }^{2,4}$, Shaojuan Qiu ${ }^{1,2}$, \\ Shuming Zhong ${ }^{3}$, Lianping Zhao ${ }^{1,5}$, Feng Chen ${ }^{1,2}$, Shunkai Lai ${ }^{3}$, Zhangzhang Qi ${ }^{1,2}$, \\ Li Huang ${ }^{1,2 *}$ and Ying Wang ${ }^{1,2 *}$

\begin{abstract}
${ }^{1}$ Medical Imaging Center, First Affiliated Hospital of Jinan University, Guangzhou, China, ${ }^{2}$ Institute of Molecular and Functional Imaging, Jinan University, Guangzhou, China, ${ }^{3}$ Department of Psychiatry, First Affiliated Hospital of Jinan University, Guangzhou, China, ${ }^{4}$ The Sixth Affiliated Hospital of Sun Yat-sen University, Guangzhou, China, ${ }^{5}$ Department of Radiology, Gansu Provincial Hospital, Lanzhou, China
\end{abstract}

OPEN ACCESS

Edited by:

Chao-Gan Yan,

Chinese Academy of Sciences, China

Reviewed by:

Qiu Jiang,

Southwest Normal University, China

Ruiwang Huang,

South China Normal University, China

*Correspondence:

Li Huang

cjr.huangli@vip.163.com

Ying Wang

johneil@vip.sina.com

tThese authors have contributed equally to this work

Specialty section:

This article was submitted to Neuroimaging and Stimulation,

a section of the journal

Frontiers in Psychiatry

Received: 18 May 2018 Accepted: 03 December 2018 Published: 18 December 2018

Citation:

Luo X, Chen G, Jia Y, Gong J, Qiu S, Zhong S, Zhao L, Chen F, Lai S, Qi Z,

Huang $L$ and Wang $Y$ (2018)

Disrupted Cerebellar Connectivity With the Central Executive Network and the Default-Mode Network in

Unmedicated Bipolar II Disorder.

Front. Psychiatry 9:705

doi: 10.3389/fpsyt.2018.00705
Objective: Bipolar disorder (BD) is a common psychiatric disease. Although structural and functional abnormalities of the cerebellum in BD patients have been reported by recent neuroimaging studies, the cerebellar-cerebral functional connectivity (FC) has not yet been examined. The present study aims to investigate the $\mathrm{FC}$ between the cerebellum and cerebrum, particularly the central executive network (CEN) and the default-mode network (DMN) in bipolar II disorder (BD II).

Methods: Ninety-four patients with unmedicated BD II depression and 100 healthy controls (HCs) underwent the resting-state functional magnetic resonance imaging. Seed-based connectivity analyses were performed using cerebellar seeds previously identified as being involved in the CEN (bilateral Crus la) and DMN (bilateral Crus lb).

Results: Compared with HCs, BD II depression patients appeared decreased FC in the right Crus la-left dorsal lateral prefrontal cortex (dIPFC) and -left anterior cingulate cortex (ACC), the right Crus lb-left medial prefrontal cortex (mPFC), -left middle temporal gyrus (MTG), and -left inferior temporal gyrus (ITG). No altered FC between the left Crus la or Crus $\mathrm{Ib}$ and the cerebral regions was found.

Conclusions: Patients with BD II depression showed disrupted FC between the cerebellum and the CEN (mainly in the left dIPFC and ACC) and DMN (mainly in the left mPFC and temporal lobe), suggesting the significant role of the cerebellum-CEN and -DMN connectivity in the pathogenesis of BD.

Keywords: bipolar disorder, functional magnetic resonance imaging, cerebellar-cerebral functional connectivity, default-mode network, central executive network

\section{INTRODUCTION}

Bipolar disorder $(\mathrm{BD})$ is a chronic, severe and fluctuating psychiatric disease and receives widespread attention due to its various clinical manifestation, complicated course, and difficulty in treatment. Though $\mathrm{BD}$ consists of recurring episodes of mania and depression, the most common manifestation is the depressive episode, which results in a misdiagnosis as a major depressive 
disorder (MDD), bringing about mistreatment, huge medical costs, and poor clinical outcome. Despite being a common and important psychiatric illness, the specific pathogenesis of $\mathrm{BD}$ remains unclear. The proposal that the cerebellum plays a significant role in the pathophysiological mechanisms of $\mathrm{BD}$ was raised these years (1). Some neuroimaging studies have demonstrated structural and functional abnormalities of the cerebellum in BD (2-4).

The cerebellum has traditionally been regarded as an important role in motor control, posture coordination, and linguistic processing. However, recently, some evidence from literatures highlight an association between the cerebellum and higher-order functions, such as non-motor cognition as well as emotion (5-7). Anatomically, the cerebellum of normal human has been divided into ten lobules named I-X, grouped as the anterior lobe (lobules I-V); posterior lobe (lobules VI-IX, including Crus I and Crus II and lobule VIIb); and the flocculonodular lobe (lobule X) $(8,9)$. And it has been shown to reciprocally connect with many brain regions, like the brainstem reticular nuclei, hypothalamus, periaqueductal gray matter, amygdala, prefrontal cortex, and anterior cingulate cortex (ACC) (10-12). These connections are hypothesized to be the neural substrates for the cerebellar-cerebral functional connectivity (FC). Functionally, several resting-state functional magnetic resonance imaging (rs-fMRI) data have demonstrated FC between the cerebellar subregions and cerebral networks. For instance, using voxel-based analysis, a study has reported strong FC between lobule Crus I, Crus II, and the frontoparietal network; between lobule IX, vermal VIIIb and the defaultmode network (DMN); between lobules I-VI, VIII and the sensorimotor networks and so on in 228 normal humans (13). Performing independent component analysis in 15 normal humans, Habas et al. found that cerebellar Crus I and II mainly participated in the bilateral central executive network (CEN), lobule IX participated in the DMN, lobules V-VI participated in the sensorimotor networks, and lobule VI participated in salience network (14). Meanwhile, applying seed-based analysis in 40 normal humans, Krienen and Buckner have also found that subregions in cerebellum had FC with the CEN, DMN, and motor network (15).

The CEN was classically conceptualized as referring to many fields, including working memory, initiation, planning, inhibition, flexibility, and vigilance (16). And the DMN was suggested to subserve ongoing, or default functions of the brain (17). These two networks are recognized as 2 core neurocognitive networks (18), showing dysfunctional connectivity in neuropsychiatric disorders, such as Alzheimer's disease, MDD, and BD (19). Moreover, several rs-fMRI studies have displayed aberrant FC between the cerebellum and the CEN and DMN in MDD (20-22), and schizophrenia (23), suggesting that the cerebellum participated in the pathogenesis of psychiatric diseases. However, only one study paid close attention to the cerebellar FC in $\mathrm{BD}$, which used cerebral seeds to probe the cerebral-cerebellar $\mathrm{FC}$ in $\mathrm{BD}$ with psychosis, and found cerebral-cerebellar dysconnectivity in selective networks, such as the somatomotor, ventral attention, salience, and frontoparietal networks (24).
$\mathrm{BD}$ has two main subtypes, bipolar I disorder (BD I), and bipolar II disorder (BD II), which present different affective states and personality characteristics. According to the Diagnostic and Statistical Manual of Mental Disorders, Fifth Edition (known as DSM-V), BD I have a manic episode while BD II have a hypomanic episode. Though patients with $\mathrm{BD}$ II have less severe intensity, several studies suggest that patients with $\mathrm{BD}$ II may have a more chronic course, higher frequency of depressive episodes and comorbidity, more suicidal behavior, and rapid cycling than patients with BD I (25-29). There are reports about the genetic $(28,30,31)$, metabolic (32), and electrophysiological $(25,33,34)$ differences between BD II and BD I. And neuroimaging studies have shown the differences in structure (29, 35-39), and task-based function (40-42) between BD II and BD I. However, most neuroimaging studies, especially rs-fMRI studies, recruited heterogeneous samples of varying BD subtypes that included BD I, BD II. To date, the neurobiology of BD II has been poorly investigated. No prior study has researched the FC between cerebellum and cerebrum in BD II.

In the present study, we collected rs-fMRI data from patients with unmedicated BD II depression and healthy controls (HCs) at relatively large sample scale. To investigate the intrinsic cerebellar-cerebral FC in the CEN and DMN in BD II depression patients and HCs, seed-based correlation analyses were conducted. Cerebellar seeds were used to identify the $\mathrm{CEN}$, and DMN, which were demonstrated to have a cerebralcerebellar connection by previous studies $(7,15,21,22)$. We hypothesized that BD II depression patients would have aberrant $\mathrm{FC}$ in the cerebellum -CEN and -DMN.

\section{METHODS AND MATERIALS}

\section{Subjects}

A total of 200 individuals ranging in age from 18 to 55 years participated in this study, including 97 currently depressed adults diagnosed with BD II and 103 HCs. The patients were recruited from the psychiatry department, First Affiliated Hospital of Jinan University, Guangzhou, China. All patients met DSM-V criteria for BD II according to the diagnostic assessment by the Structured Clinical Interview for DSM-V Patient Edition (SCID-P). The clinical state was assessed by using the 24item Hamilton Depression Rating Scale (HDRS) and the Young Mania Rating Scale (YMRS) during the 3-day period prior to the imaging session. To be eligible for the study, all patients should have a total HDRS-24 score of $>21$ and total YMRS score of $<7$. At the time of testing, all patients were either medication-naïve or were not medicated for at least 6 months. The exclusion criteria included the presence of (a) any other AxisI psychiatric disorders, (b) a history of electroconvulsive therapy, (c) a history of organic brain disorder, mental retardation, neurological disorders, (d) pregnancy, alcohol/substance abuse or dependence, or any presence of a concurrent and major physical illness, (e) any contraindication to MRI scanning. In addition, 103 right-handed HCs were recruited through local advertisements. To rule out the presence of a current or past history of any psychiatric illness, HCs were carefully screened through a diagnostic interview-the Structured Clinical Interview 
for DSM-V Non-patient Edition (SCID-NP). Further exclusion criteria for HCs were any history of a current or past significant medical or neurological illness, psychiatric illness in first-degree relatives.

This study was approved by the Ethics Committee of First Affiliated Hospital of Jinan University, Guangzhou, China, and signed a written informed consent for each participant after a full explanation of the study. Two senior clinical psychiatrists confirmed that all subjects had the ability to consent to participate in the examination.

\section{MRI Data Acquisition}

Imaging was performed on a GE Discovery MR750 3.0 T System with an 8-channel phased array head coil. Subjects were scanned in a supine, head-first position with symmetrically placed cushions on both sides of the head to decrease motion. Before the scanning, each participant was repeatedly instructed to relax with their eyes closed without falling asleep; after the experiment, only participants who confirmed that they had not fallen asleep were included; otherwise, he/she was excluded.

The rs-fMRI data were acquired using gradient-echo echoplanar imaging sequence with the following parameters: time repetition (TR)/time echo $(\mathrm{TE})=2,000 / 25 \mathrm{~ms}$, flip angle $=90^{\circ}$, voxel size $=3.75 \times 3.75 \times 3 \mathrm{~mm}^{3}$, field of view $(\mathrm{FOV})=240$ $\times 240 \mathrm{~mm}$, matrix $=64 \times 64$, slice thickness $/$ gap $=3.0 / 1.0 \mathrm{~mm}$, 35 axial slices covering the whole-brain, and 210 volumes acquired in $7 \mathrm{~min}$. In addition, a three-dimensional brain volume imaging (3D-BRAVO) sequence covering the whole brain was used for structural data acquisition with: $\mathrm{TR} / \mathrm{TE}=8.2 / 3.2 \mathrm{~ms}$, flip angle $=12^{\circ}$, bandwidth $=31.25 \mathrm{~Hz}$, slice thickness/gap $=$ $1.0 / 0 \mathrm{~mm}$ matrix $=256 \times 256, \mathrm{FOV}=240 \times 240 \mathrm{~mm}, \mathrm{NEX}=$ 1 , and acquisition time $=3 \mathrm{~min} 45 \mathrm{~s}$. Routine MRI examination images were also collected for excluding anatomic abnormality. All participants were found by two experienced radiologists to confirm no brain structural abnormalities.

\section{Data Preprocessing}

The preprocessing was carried out using Data Processing and Analysis of Brain Imaging (DPABI, http://rfmri.org/DPABI), which is based on Statistical Parametric Mapping (SPM12, http://www.fil.ion.ucl.ac.uk/spm/). For each subject, the first 10 images of the rs-fMRI dataset were excluded to ensure steadystate longitudinal magnetization. The remaining 200 images were first slice-time corrected and then were realigned to the first image for correcting for inter-TR head motion. This realignment correction provided a record of the head motion within the rs-fMRI scan. All subjects should have no more than $2 \mathrm{~mm}$ maximum displacement in any plane, $0.2 \mathrm{~mm}$ in mean frame-wise displacement (FD) (43), and $2^{\circ}$ of angular motion. The individual T1 structural images were segmented (white matter, gray matter, and cerebrospinal fluid) using Segmentation toolbox. Then, the Diffeomorphic Anatomical Registration Through Exponentiated Lie algebra (DARTEL) toolbox was used to create a study specific template for the accurate normalization. Then, resting-state functional images were coregistered to the structural images and transformed into standard Montreal Neurological Institute (MNI) space, resliced to a voxel size of $3 \times 3 \times 3 \mathrm{~mm}^{3}$ resolution and smoothed using a $4 \mathrm{~mm}$ full width at half maximum (FWHM) Gaussian kernel. Furthermore, the data were removed linear trend and passed through band-pass filtered of $0.01-0.1 \mathrm{~Hz}$. The Friston24-parameter model of head motion (including the 6 head motion parameters, 6 head motion parameters one time point before, and the 12 corresponding squared items) (44) was chosen based on prior work (45). Several spurious covariates and their temporal derivatives were then regressed out from the time course of each voxel, including the signals of the brain global mean, white matter, and cerebrospinal fluid as well as the Friston24 parameters of head motion.

\section{FC Analysis}

Two previous seed-based rs-fMRI studies have reported that the left cerebellar Crus Ia (MNI coordinates: -12, -78, -28) and right cerebellar Crus Ia (MNI coordinates: 12, -78, -28) participated in the CEN, and the left cerebellar Crus Ib (MNI coordinates: $-32,-76,-34)$ and right cerebellar Crus Ib (MNI coordinates: $34,-80,-36)$ participated in the DMN in major depressive disorder $(21,22)$. It was noticed that the bilateral Crus Ib involved in the DMN were asymmetric. These two seeds were defined by Krienen and Buckner in a study that used frontal seeds to investigate the FC between the cerebrum and cerebellum in normal humans (15). They found that the DMN (mPFC) was strongly connected with the bilateral Crus Ib. Thus, we chose these four seeds with a radius of $6 \mathrm{~mm}$ to examine the FC between the cerebellum and the CEN and the DMN (Figure 1). The seed point reference time series of each seed was extracted by averaging the time series of voxels of each ROI. For each subject, correlation maps were produced by computing the Pearson's correlation coefficients between the time series of the seeds and all the other brain voxels. To improve the normality, the obtained correlation maps were then converted to $z$-values maps using Fisher's $r$-to- $z$ transformation. For all the subjects, four $z$-score maps which represented the intrinsic FC of the four cerebellar seeds were finally obtained.

\section{Statistical Analysis}

The two final group of participants included in this study were 94 patients with BD II (mean age, SD, gender ration) and 100 HCs. Independent-sample $t$-tests and $\chi^{2}$ tests were used to compare the demographic data between the BD II and HCs groups with SPSS 17.0 software (SPSS, Chicago, IL, USA). All tests were two-tailed, and $p<0.05$ were considered statistically significant.

The one-sample $t$-test was performed on $\mathrm{z}$-score maps to demonstrate the within-group FC spatial distribution of each cerebellar seed for the BD patients and HCs. Then, the twosample $t$-test was performed to assess the significant differences of the whole brain FC for each cerebellar seed between BD patients and HCs within the union mask of one-sample $t$ test results of both groups. Age, gender, education level, and the mean FD were included as nuisance covariates in the group comparisons. Statistical maps were thresholded using permutation test (PT) as implemented in Permutation Analysis of Linear Models (PALM) (46) and integrated into DPABI. The threshold-free cluster enhancement (TFCE) and voxel-wise 


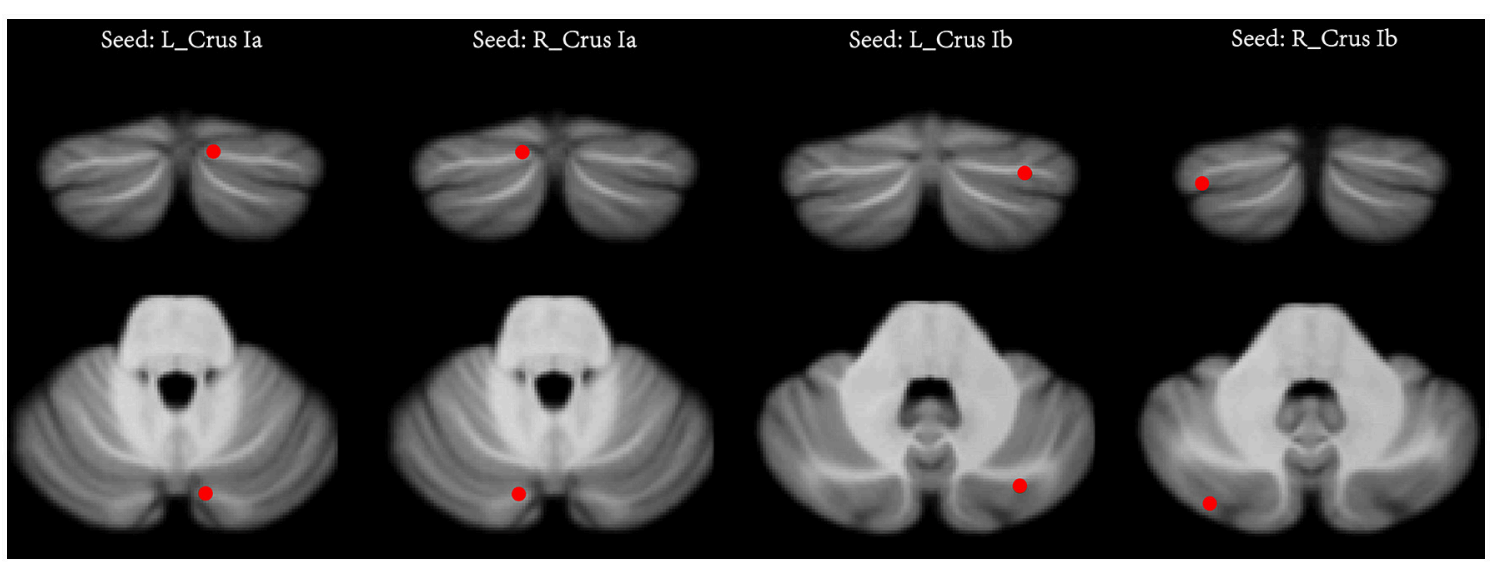

FIGURE 1 | The seeds of the cerebellum. In each hemisphere, two seeds were defined, including the Crus la and Crus lb. L (R), left (right) hemisphere.

correction (VOX) with PT were tested at two-tailed $p<0.05$ for multiple comparisons. The number of permutations was set at 1,000 .

Once statistically significant group differences were observed in any brain FC, we calculated the Pearson correlation coefficients between the clinical variables and FC values in BD II group. These clinical variables included onset age of illness, number of episodes, duration of illness, HDRS score, and YMRS score.

\section{RESULTS}

\section{Demographic Information}

Table 1 shows the demographic and clinical information of all study participants. Three patients with BD II and three HCs were discarded from further analyses owing to excessive head movement during the image acquisition. Finally, the participants included 94 BD II patients and 100 HCs. The two groups have no significant differences in sex, age, years of education, and FD parameter.

\section{FC Analysis Within-Group}

One-sample $t$-tests displayed the within-group FC patterns in the $\mathrm{BD}$ II and HCs group. Within the HCs group, the FC spatial distribution of the bilateral cerebellar Crus Ia is mainly located in the CEN, including the bilateral dIPFC, ACC, and superior parietal cortext. And the FC spatial distribution of the bilateral cerebellar Crus Ib is mainly located in the DMN, including the bilateral $\mathrm{mPFC}$, posterior cingulate cortex, precuneus, inferior parietal lobule, middle temporal gyrus (MTG), and inferior temporal gyrus (ITG). Visual inspection indicated that FC patterns of the bilateral cerebellar Crus Ia and Crus Ib in the BD II group were similar to those of the HCs group.

\section{FC Analysis Between-Group}

For the CEN, the patients with BD II displayed decreased FC in the right Crus Ia-left dlPFC, and -left ACC compared with HCs (Table 2, Figure 2). For the DMN, the patients with BD II displayed decreased FC in the right Crus Ib-left mPFC (ventral
TABLE 1 | Demographic and Clinical Data in BD || patients and HCs.

\begin{tabular}{lccc}
\hline Variables & BD II & HCs & P-Value \\
\hline No. of participants & 94 & 100 & \\
Gender (M: F) & $51: 43$ & $45: 55$ & $0.198^{\star \star}$ \\
Age (years) & $27.18 \pm 9.15$ & $28.32 \pm 8.95$ & $0.383^{\star}$ \\
Years of education & $14.06 \pm 2.79$ & $14.72 \pm 2.76$ & $0.101^{\star}$ \\
Age at onset (years) & $22.36 \pm 8.94$ & NA & \\
No. of episodes & $3.01 \pm 1.45$ & NA & \\
Duration of illness (months) & $47.11 \pm 60.51$ & NA & \\
24-item HDRS score (points) & $26.02 \pm 6.80$ & NA & \\
YMRS score (points) & $3.98 \pm 5.27$ & NA & \\
FD values (mm) & $0.08 \pm 0.04$ & $0.08 \pm 0.04$ & $0.497^{\star}$ \\
\hline
\end{tabular}

Unless otherwise indicated, data are means \pm standard deviation. BD, bipolar disorder; HCs, healthy controls; HDRS, Hamilton Depression Rating Scale; YMRS, Young Mania Rating Scale; FD, framewise displacement for in-scanner head motion; NA, not applicable. ${ }^{\star *} P$-value for sex distribution obtained by $x^{2}$ test. ${ }^{*} P$-values obtained by independent sample tests.

mPFC, vmPFC), -left MTG, and -left ITG (fusiform gyrus) compared with HCs (Table 2, Figure 2). There was no aberrant FC between the left Crus Ia or Crus Ib and the cerebral regions in BD II patients compared to the HCs. In addition, there was no increased FC in the BD II group relative to the $\mathrm{HCs}$ group. No significant correlations between the $\mathrm{FC}$ values in these regions and any clinical measure (including onset age of illness, number of episodes, duration of illness, HDRS, YMRS scores) in the BD II patients were found.

\section{DISCUSSION}

Using well-defined cerebellar seeds, we investigated the alteration of cerebellar-cerebral connectivity in BD II patients and found evidence of abnormalities in selective networks. Particularly, we found decreased cerebellar-cerebral FC in the CEN and DMN, particularly decreased Crus Ia-dlPFC, and ACC connectivity, and Crus Ib-mPFC, MTG, and ITG connectivity in unmedicated 
TABLE 2 | Differences of cerebellar-cerebral FC between BD || patients and HCs.

\begin{tabular}{|c|c|c|c|c|c|c|c|c|}
\hline \multirow[t]{2}{*}{ Cerebellar seeds } & \multirow[t]{2}{*}{ Significant regions } & \multirow[t]{2}{*}{$t$ value } & \multirow[t]{2}{*}{ Cluster size $\left(\mathrm{mm}^{3}\right)$} & \multirow[t]{2}{*}{ side } & \multicolumn{3}{|c|}{ MNI } & \multirow[t]{2}{*}{ Brodmann area } \\
\hline & & & & & $\mathbf{x}$ & y & $\mathbf{z}$ & \\
\hline \multicolumn{9}{|l|}{$\mathrm{BD}<\mathrm{HCs}$} \\
\hline \multirow[t]{3}{*}{ R Crus la } & dIPFC & -4.7902 & 810 & $L$ & -48 & 27 & 18 & 9,46 \\
\hline & dIPFC & -3.8646 & 2160 & $L$ & -33 & 6 & 48 & 9 \\
\hline & ACC & -4.4720 & 81 & $L$ & -6 & 33 & 33 & 32 \\
\hline \multirow[t]{4}{*}{ R Crus Ib } & vmPFC & -3.5598 & 324 & $L$ & -15 & 57 & 12 & 10 \\
\hline & mPFC & -4.4338 & 1161 & $L$ & -24 & 42 & 45 & 8 \\
\hline & MTG & -4.3466 & 1242 & $L$ & -69 & -39 & -9 & 21 \\
\hline & ITG (fusiform gyrus) & -3.9390 & 594 & $\mathrm{~L}$ & -54 & -15 & -30 & 20 \\
\hline
\end{tabular}

FC, functional connectivity; BD, bipolar disorder; HCs, healthy controls; MNI, Montreal Neurological Institute; R, right; L, left; dIPFC, dorsal lateral prefrontal cortext; ACC, anterior cingulate cortext; vMPFC, ventral medial prefrontal cortex; MPFC, medial prefrontal cortex; MTG, middle temporal gyrus; ITG, inferior temporal gyrus.

patients with bipolar II depression. This is the first study to report abnormal cerebellar connectivity with the CEN and DMN in patients with bipolar II depression.

The dIPFC and ACC are recognized as important regions within the CEN and are significant roles in maintaining cognition and emotion control (47-49). In this study, we found reduced FC within right Crus Ia-left dlPFC, and right Crus Ia-left ACC in patients with bipolar II depression, suggesting the impaired FC between the cerebellum and the CEN. Using seed-based connectivity analyses, a recent rs-fMRI found reduced cerebrocerebellar FC in frontoparietal control networks in psychotic BD (24). Another rs-fMRI study reported decreased FC between the cerebellum and the dIPFC in patients with geriatric depression (21). Several studies demonstrated structural and functional abnormality of the dlPFC in BD. For example, several structural MRI studies reported reduced cortical thickness $(50,51)$ and gray matter volume (52) in the dlPFC in bipolar depression. Additionally, some rs-fMRI studies showed reduced FC between the dlPFC and mPFC (53), right temporal gyrus (54), left insula (55), and amygdala (56) in BD patients using seed-based approach or global brain connectivity method. Furthermore, task-based fMRI studies revealed that BD depressed patients showed decreased activation in the dlPFC and cerebellum during n-back tasks $(57,58)$. A previous PET study found that decreased dlPFC metabolism was associated with sustained attention deficits in depressed adults with BD (59). The ACC is a key region implicated in mood regulation (60) and might be a possible trait feature of $\mathrm{BD}(61,62)$. An rs-fMRI study revealed decreased FC between the defined cingulo-opercular network (included the dorsal ACC attributed to cognition) and cerebellar network in BD (23). In addition, performing an emotion processing task, $\mathrm{BD}$ patients showed decreased activation in the ACC compared to HCs $(63,64)$. Therefore, disrupt connectivity of the cerebellar Crus Ia-dlPFC and -ACC may be associated with dysfunctional cognition and emotion control in BD II patients.

The DMN was suggested to subserve ongoing, or default functions of the brain such as self-referential mental activity, episodic memory retrieval, inner speech, mental images, emotions, and planning future events $(17,65)$. In this study, patients with BD II depression showed decreased FC in the cerebellum connected to the mPFC (mainly in the vmPFC). Using seed-based connectivity analyses, Alalade et al. reported that depression patients had reduced FC between the cerebellar seeds and the vmPFC (21). The mPFC is a core hub of the DMN in which abnormalities have been reported in BD (66). Structural MRI found decreased gray matter volume and density predominantly in the vmPFC in BD patients (67-69). And in rs-fMRI studies, Wang et al. found diminished functional connectivity strength (70) and voxel-mirrored homotopic connectivity (71) in the mPFC in non-medicine $\mathrm{BD}$ II patients. Using independent component analysis, Öngür et al. reported reduced DMN connectivity in the vmPFC in BD patients (72). Moreover, several studies demonstrated that ruminative self-focus was associated with the aberrant FC/activation in the mPFC in depression (7376). A task-based fMRI study revealed that $\mathrm{BD}$ patients have decreased FC between the vmPFC and the head of the caudate, performing a self-reflection task (77). Taken together, our finding of the aberrant Crus Ib-mPFC connectivity may be involved in the abnormality of self-referential mental activity in $\mathrm{BD}$ patients.

The temporal gyrus is suggested to be in involved in several cognitive processing, including visual perception (ITG), language and semantic memory processing (MTG), sensory integration (78). In this study, the depressed BD II patients showed reduced FC between the right Crus $\mathrm{Ib}$ and left temporal lobe, including the MTG and ITG. A structural MRI study found reduced cortical thickness in the left MTG, ITG, and vmPFC in BD II patients (79). Two rs-fMRI studies demonstrated that the $\mathrm{BD}$ depression patients showed a reduction of functional connectivity strength in the left MTG (80) and reduction of the voxel-mirrored homotopic connectivity in the cerebellum and fusiform (81). Additionally, task-based fMRI studies found reduced activation in the MTG in BD II depression patients during an n-back task (82), and reduced activation in the MTG and ITG in BD I during an attentional task (83) and visuospatial processing task (84). Thus, these studies, united with our findings, suggest that the reduced FC within the Crus IbMTG and -ITG may be associated with disruption of cognition in $\mathrm{BD}$. 


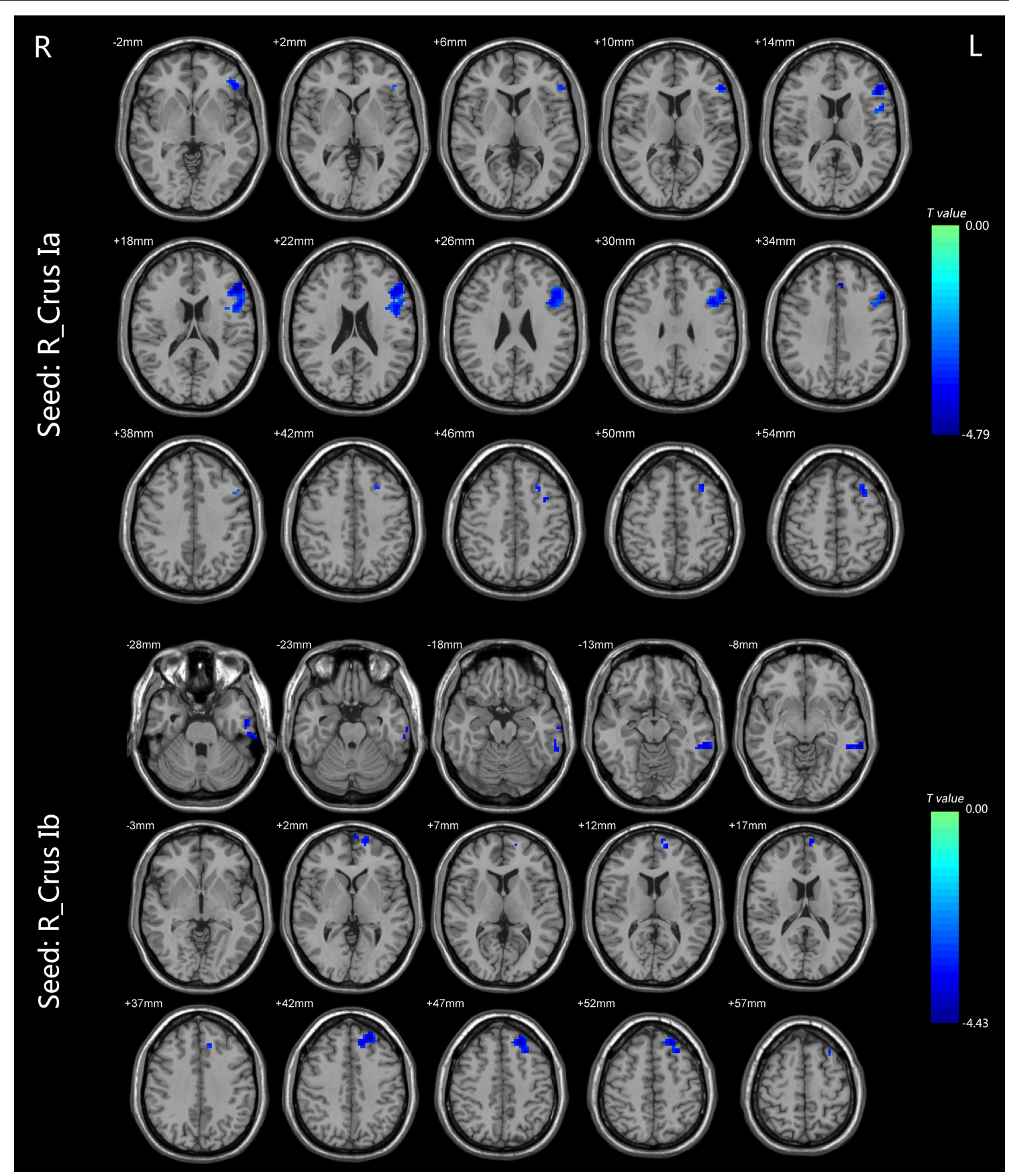

FIGURE 2 | The FC differences between the BD patients and HCs $(p<0.05$, TFCE corrected). For the CEN, the patients with BD II displayed decreased FC in the right Crus la-left dIPFC and left ACC compared with HCs. For the DMN, the patients with BD II displayed decreased FC in the right Crus Ib-left mPFC (vmPFC), left MTG, and left ITG (fusiform gyrus) compared with HCs. Shades of blue denoted decreased FC in the BD group compared with HCs group. The color bars indicate the $t$-values. FC, functional connectivity; BD, bipolar disorder; HCs, healthy controls; TFCE, the threshold-free cluster enhancement; CEN, central executive network; dIPFC, dorsal lateral frontal cortext; ACC, anterior cingulate cortext; DMN, default mode network; mPFC, medial prefrontal cortex; vmPFC, ventral medial prefrontal cortex; MTG, middle temporal gyrus; ITG, inferior temporal gyrus.

What's more, unexpectedly, it's revealed that all the findings of reduced cerebellar-cerebral FC in patients appear between the right cerebellar seeds and the regions in the left cerebral hemisphere. There are pieces of evidence supporting abnormal hemisphere asymmetries in the major psychotic disorders, such as schizophrenia, MDD, and $\mathrm{BD}$. Generally, a predominant left hemisphere disturbance in schizophrenia was reported by psychophysiological, neuropsychological, and neuroimaging 
studies (85-89). Electrophysiology, rs-fMRI and task-based fMRI studies showed a predominantly left-sided reduction of activity in the PFC in depression (90-94). And several studies found that patients with left hemisphere damage or lesions tended to appear depressive symptoms (95-97), while patients with right hemisphere damage or lesions tended to appear manic symptoms $(97,98)$. Two studies founded lower activation in the left hemisphere than in the right hemisphere in the $\mathrm{BD}$ depression while the opposite in the $\operatorname{BD}$ mania $(99,100)$, which is consistent with our finding of asymmetric left hemisphere disturbance in BD II depression. This phenomenon was reported to be associated with a high percentage of dopaminergic synapses in the left hemisphere, and the dopamine is essential in regulating motor activity, biological substrate of positive emotions and approaching behavior (99). Thus, the hemispheric asymmetry aberrance may be a significant biological substrate in BD.

There were several strengths in our study. First, we recruited a large number of participants of $94 \mathrm{BD}$ II patients and $100 \mathrm{HCs}$, and the samples of non-medicine patients with BD II depression are homogeneous, while most previous rs-fMRI studies recruited heterogeneous samples of varying BD subtypes including BD I and BD II. Second, we noted permutation test with TFCE for multiple comparison correction in between-group contrast. A recent study demonstrated that the PT with TFCE, which is a strict multiple comparison correction strategy, was best able to reach the balance between family-wise error rate and testretest reliability/replicability (101). However, there are also some potential limitations that should be taken into consideration. First, without a group of patients with $\mathrm{BD}$ in a euthymic episode, it is still not clear whether aberrant cerebellar-cerebral $\mathrm{FC}$ is specific to the depression episode of $\mathrm{BD}$ or shared by all episodes of the disease. Second, BD patients didn't participate in cognitive test scales, such as the Wisconsin Card Sorting Test, Digit Span Test, and Stroop Color Word Test. Further studies of the correlations between the impaired FC values and cognitive function are needed to support our speculations that

\section{REFERENCES}

1. Minichino A, Bersani FS, Trabucchi G, Albano G, Primavera M, Delle Chiaie $\mathrm{R}$, et al. The role of cerebellum in unipolar and bipolar depression: a review of the main neurobiological findings. Riv Psichiatr. (2014) 49:124-31. doi: 10.1708/1551.16907

2. Zhao L, Wang Y, Jia Y, Zhong S, Sun Y, Zhou Z, et al. Cerebellar microstructural abnormalities in bipolar depression and unipolar depression: a diffusion kurtosis and perfusion imaging study. J. Affect. Disord. (2016) 195:21-31. doi: 10.1016/j.jad.2016.01.042

3. Dolan RJ, Bench CJ, Brown RG, Scott LC, Friston KJ, Frackowiak RS. Regional cerebral blood flow abnormalities in depressed patients with cognitive impairment. J Neurol Neurosurg Psychiatry (1992) 55:768-73. doi: 10.1136/jnnp.55.9.768

4. Liu CH, Li F, Li SF, Wang YJ, Tie CL, Wu HY, et al. Abnormal baseline brain activity in bipolar depression: a resting state functional magnetic resonance imaging study. Psychiatr Res. (2012) 203:175-9. doi: 10.1016/j.pscychresns.2012.02.007

5. Kirschen MP, Davis-Ratner MS, Milner MW, Chen SHA, SchraedleyDesmond P, Fisher PG, et al. Verbal memory impairments in children the cerebellum-CEN and -DMN FC is involved in the cognition deficits in BD. Third, we didn't analyses the morphology characteristics of the $\mathrm{BD}$ patients, which should be considered and combined with our findings to provide more reliable imaging evidence in the pathogenesis of BD. Forth, further studies should be taken to investigate the differences of FC between BD II and BD I. Finally, we confirmed that the participants had not fallen asleep via their self-report, which could not ensure that each participant had not actually fallen asleep during the period of scanning.

\section{CONCLUSION}

Patients with BD II depression showed disrupted FC between the cerebellum and the CEN (mainly in the left dlPFC and ACC) and DMN (mainly in the left mPFC, and temporal lobe), suggesting the significant role of the cerebellum-CEN and -DMN connectivity in the pathogenesis of BD.

\section{AUTHOR CONTRIBUTIONS}

XL, LH, and YW design the study; GC, YJ, JG, SQ, SZ, LZ, FC, $\mathrm{SL}$, and ZQ contribute to data acquisition; GC contribute to data analysis; XL, GC, LH, and YW write the manuscript. All authors contributed to and have approved the final manuscript.

\section{FUNDING}

The study was supported by grants from the National Natural Science Foundation of China (81671670, 81501456, and 81471650); Planned Science and Technology Project of Guangdong Province, China (2014B020212022); Planned Science and Technology Project of Guangzhou, China (1563000653, 201508020004, 201604020007, and 201604020184). The funding organizations played no further role in study design, data collection, analysis and interpretation, and paper writing. after cerebellar tumor resection. Behav Neurol. (2008) 20:39-53. doi: $10.1155 / 2008 / 817253$

6. Laricchiuta D, Petrosini L, Picerni E, Cutuli D, Iorio M, Chiapponi C, et al. The embodied emotion in cerebellum: a neuroimaging study of alexithymia. Brain Struct Funct. (2015) 220:2275-87. doi: 10.1007/s00429-014-0790-0

7. Stoodley CJ, Schmahmann JD. Functional topography in the human cerebellum: a meta-analysis of neuroimaging studies. Neuroimage (2009) 44:489-501. doi: 10.1016/j.neuroimage.2008.08.039

8. Stoodley CJ, Valera EM, Schmahmann JD. Functional topography of the cerebellum for motor and cognitive tasks: an fMRI study. Neuroimage (2012) 59:1560-70. doi: 10.1016/j.neuroimage.2011.08.065

9. Schmahmann JD, Doyon J, McDonald D, Holmes C, Lavoie K, Hurwitz AS, et al. Three-dimensional MRI atlas of the human cerebellum in proportional stereotaxic space. Neuroimage (1999) 10:233-60. doi: 10.1006/nimg.1999.0459

10. Vilensky JA, van Hoesen GW. Corticopontine projections from the cingulate cortex in the rhesus monkey. Brain Res. (1981) 205:391-5. doi: 10.1016/0006-8993(81)90348-6

11. Haines DE, Dietrichs E, Sowa TE. Hypothalamo-cerebellar and cerebellohypothalamic pathways: a review and hypothesis concerning cerebellar 
circuits which may influence autonomic centers affective behavior. Brain, Behav Evol. (1984) 24:198-220. doi: 10.1159/000121317

12. Heath RG, Dempesy CW, Fontana CJ, Myers WA. Cerebellar stimulation: effects on septal region, hippocampus, and amygdala of cats and rats. Biol Psychiatry (1978) 13:501-29.

13. Sang L, Qin W, Liu Y, Han W, Zhang YT, Jiang TZ, et al. Resting-state functional connectivity of the vermal and hemispheric subregions of the cerebellum with both the cerebral cortical networks and subcortical structures. Neuroimage (2012) 61:1213-25. doi: 10.1016/j.neuroimage.2012.04.011

14. Habas C, Kamdar N, Nguyen D, Prater K, Beckmann CF, Menon V, et al. Distinct cerebellar contributions to intrinsic connectivity networks. $J$ Neurosci. (2009) 29:8586-94. doi: 10.1523/JNEUROSCI.1868-09.2009

15. Krienen FM, Buckner RL. Segregated fronto-cerebellar circuits revealed by intrinsic functional connectivity. Cereb Cortex (2009) 19:2485-97. doi: 10.1093/cercor/bhp135

16. Smith SM, Fox PT, Miller KL, Glahn DC, Fox PM, Mackay CE, et al. Correspondence of the brain's functional architecture during activation and rest. Proc Natl Acad Sci USA. (2009) 106:13040-5. doi: 10.1073/pnas.0905267106

17. Gusnard DA, Raichle ME, Raichle ME. Searching for a baseline: functional imaging and the resting human brain. Nat Rev Neurosci. (2001) 2:685-94. doi: $10.1038 / 35094500$

18. Menon V. Large-scale brain networks and psychopathology: a unifying triple network model. Trends Cogn Sci. (2011) 15:483-506. doi: 10.1016/j.tics.2011.08.003

19. Sha Z, Xia M, Lin Q, Cao M, Tang Y, Xu K, et al. Meta-connectomic analysis reveals commonly disrupted functional architectures in network modules and connectors across brain disorders. Cereb Cortex (2017) 28:4179-94. doi: $10.1093 /$ cercor/bhx273

20. Liu L, Zeng LL, Li YM, Ma QM, Li BJ, Shen H, et al. Altered cerebellar functional connectivity with intrinsic connectivity networks in adults with major depressive disorder. PLoS ONE (2012) 7:e39516. doi: 10.1371/journal.pone.0039516

21. Alalade E, Denny K, Potter G, Steffens D, Wang LH. Altered Cerebellarcerebral functional connectivity in geriatric depression. PLoS ONE (2011) 6:e20035. doi: 10.1371/journal.pone.0020035

22. Guo WB, Liu F, Xue ZM, Gao KM, Liu ZN, Xiao CQ, et al. Abnormal restingstate cerebellar-cerebral functional connectivity in treatment-resistant depression and treatment sensitive depression. Prog Neuropsychopharmacol Biol Psychiatry (2013) 44:51-7. doi: 10.1016/j.pnpbp.2013.01.010

23. Mamah D, Barch DM, Repovs G. Resting state functional connectivity of five neural networks in bipolar disorder and schizophrenia. J Affect Dis. (2013) 150:601-9. doi: 10.1016/j.jad.2013.01.051

24. Shinn AK, Roh YS, Ravichandran CT, Baker JT, Ongur D, Cohen BM. Aberrant cerebellar connectivity in bipolar disorder with psychosis. Biol Psychiatry Cogn Neurosci Neuroimaging (2017) 2:438-48. doi: 10.1016/j.bpsc.2016.07.002

25. Ma G, Wang C, Jia Y, Wang J, Zhang B, Shen C, et al. Electrocardiographic and electrooculographic responses to external emotions and their transitions in bipolar i and ii disorders. Int J Environ Res Public Health (2018) 15:884. doi: 10.3390/ijerph15050884

26. Baek JH, Park DY, Choi J, Kim JS, Choi JS, Ha K, et al. Differences between bipolar I and bipolar II disorders in clinical features, comorbidity, and family history. J Affect Disord. (2011) 131:59-67. doi: 10.1016/j.jad.2010.11.020

27. Dell'Osso B, Shah S, Do D, Yuen LD, Hooshmand F, Wang PW, et al. American tertiary clinic-referred bipolar II disorder versus bipolar I disorder associated with hastened depressive recurrence. Int J Bipolar Disord. (2017) 5:2. doi: 10.1186/s40345-017-0072-x

28. Vieta E, Suppes T. Bipolar II disorder: arguments for and against a distinct diagnostic entity. Bipolar Disord. (2008) 10:163-78. doi: 10.1111/j.1399-5618.2007.00561.x

29. Ha TH, Her JY, Kim JH, Chang JS, Cho HS, Ha K. Similarities and differences of white matter connectivity and water diffusivity in bipolar I and II disorder. Neurosci Lett. (2011) 505:150-4. doi: 10.1016/j.neulet.2011.10.009

30. Lee SY, Chen SL, Chang YH, Chen SH, Chu CH, Huang SY, et al. The ALDH2 and DRD2/ANKK1 genes interacted in bipolar II but not bipolar I disorder. Pharmacogenet Genomics (2010) 20:500-6. doi: 10.1097/FPC.0b013e32833caa2b

31. Lee SY, Chen SL, Chen SH, Huang SY, Tzeng NS, Chang YH, et al. The COMT and DRD3 genes interacted in bipolar I but not bipolar II disorder. World J Biol Psychiatry (2011) 12:385-91. doi: 10.3109/15622975.2010.505298

32. Chou YH, Wang SJ, Lin CL, Mao WC, Lee SM, Liao MH. Decreased brain serotonin transporter binding in the euthymic state of bipolar I but not bipolar II disorder: a SPECT study. Bipolar Disord. (2010) 12:312-8. doi: 10.1111/j.1399-5618.2010.00800.x

33. Zhu QS, Wang JW, Fan HY, Ma GR, Zhang BR, Shen CC, et al. Blink reflex under external emotional-stimuli in bipolar I and II disorders. Psychiatry Res. (2018) 259:520-5. doi: 10.1016/j.psychres.2017.11.020

34. Zhu QS, Wang JW, Shen CC, Fan HY, Zhang BR, Ma GR, et al. Inhibitory brainstem reflexes under external emotional-stimuli in bipolar I and II disorders. BMC Psychiatry (2017) 17:224. doi: 10.1186/s12888-017-1390-3

35. Abe C, Ekman CJ, Sellgren C, Petrovic P, Ingvar M, Landen M. Cortical thickness, volume and surface area in patients with bipolar disorder types I and II. J Psychiatr Neurosci. (2016) 41:240-50. doi: 10.1503/jpn.150093

36. Ha TH, Ha K, Kim JH, Choi JE. Regional brain gray matter abnormalities in patients with bipolar II disorder: a comparison study with bipolar I patients and healthy controls. Neurosci Lett. (2009) 456:44-8. doi: 10.1016/j.neulet.2009.03.077

37. Hauser P, Matochik J, Altshuler LL, Denicoff KD, Conrad A, Li X, et al. MRIbased measurements of temporal lobe and ventricular structures in patients with bipolar I and bipolar II disorders. J Affect Disord. (2000) 60:25-32. doi: 10.1016/S0165-0327(99)00154-8

38. Liu JX, Chen YS, Hsieh JC, Su TP, Yeh TC, Chen LF. Differences in white matter abnormalities between bipolar I and II disorders. J Affect Disord. (2010) 127:309-15. doi: 10.1016/j.jad.2010.05.026

39. Maller JJ, Thaveenthiran P, Thomson RH, McQueen S, Fitzgerald PB. Volumetric, cortical thickness and white matter integrity alterations in bipolar disorder type I and II. J Affect Disord. (2014) 169:118-27. doi: 10.1016/j.jad.2014.08.016

40. Caseras X, Murphy K, Lawrence NS, Fuentes-Claramonte P, Watts J, Jones DK, et al. Emotion regulation deficits in euthymic bipolar I versus bipolar II disorder: a functional and diffusion-tensor imaging study. Bipolar Disord. (2015) 17:461-70. doi: 10.1111/bdi.12292

41. Dell'Osso B, Cinnante C, Di Giorgio A, Cremaschi L, Palazzo MC, Cristoffanini $\mathrm{M}$, et al. Altered prefrontal cortex activity during working memory task in bipolar disorder: a functional magnetic resonance imaging study in euthymic bipolar I and II patients. J Affect Disord. (2015) 184:11622. doi: 10.1016/j.jad.2015.05.026

42. Caseras X, Lawrence NS, Murphy K, Wise RG, Phillips ML. Ventral striatum activity in response to reward: differences between bipolar i and ii disorders. Am J Psychiatry (2013) 170:533-41. doi: 10.1176/appi.ajp.2012.12020169

43. Jenkinson M, Bannister P, Brady M, Smith S. Improved optimization for the robust and accurate linear registration and motion correction of brain images. Neuroimage (2002) 17:825-41. doi: 10.1006/nimg.2002.1132

44. Friston KJ, Williams S, Howard R, Frackowiak RS, Turner R. Movementrelated effects in fMRI time-series. Magn Reson Med. (1996) 35:346-55. doi: $10.1002 / \mathrm{mrm} .1910350312$

45. Satterthwaite TD, Elliott MA, Gerraty RT, Ruparel K, Loughead J, Calkins $\mathrm{ME}$, et al. An improved framework for confound regression and filtering for control of motion artifact in the preprocessing of resting-state functional connectivity data. Neuroimage (2013) 64:240-56. doi: 10.1016/j.neuroimage.2012.08.052

46. Winkler AM, Ridgway GR, Douaud G, Nichols TE, Smith SM. Faster permutation inference in brain imaging. Neuroimage (2016) 141:502-16. doi: 10.1016/j.neuroimage.2016.05.068

47. Niendam TA, Laird AR, Ray KL, Dean YM, Glahn DC, Carter CS. Metaanalytic evidence for a superordinate cognitive control network subserving diverse executive functions. Cogn Affect Behav Neurosci. (2012) 12:241-68. doi: 10.3758/s13415-011-0083-5

48. Breukelaar IA, Antees C, Grieve SM, Foster SL, Gomes L, Williams LM, et al. Cognitive control network anatomy correlates with neurocognitive behavior: a longitudinal study. Hum Brain Mapp. (2017) 38:631-43. doi: $10.1002 / \mathrm{hbm} .23401$ 
49. Brown A, Biederman J, Valera E, Lomedico A, Aleardi M, Makris N, et al. Working memory network alterations and associated symptoms in adults with ADHD and Bipolar Disorder. J Psychiatr Res. (2012) 46:476-83. doi: 10.1016/j.jpsychires.2012.01.008

50. Lan MJ, Chhetry BT, Oquendo MA, Sublette ME, Sullivan G, Mann JJ, et al. Cortical thickness differences between bipolar depression and major depressive disorder. Bipolar Disord. (2014) 16:378-88. doi: 10.1111/bdi.12175

51. Niu MQ, Wang Y, Jia YB, Wang JJ, Zhong SM, Lin JB, et al. Common and specific abnormalities in cortical thickness in patients with major depressive and bipolar disorders. Ebiomedicine (2017) 16:162-71. doi: 10.1016/j.ebiom.2017.01.010

52. Figueroa R, Harenski K, Nicoletti M, Brambilla P, Mallinger AG, Frank E, et al. Dorsolateral prefrontal cortex abnormalities in bipolar disorder - Possible effects of lithium treatment? Biol Psychiatry (2000) 47:S103-4. doi: 10.1016/S0006-3223(00)00608-9

53. Favre P, Baciu M, Pichat C, Bougerol T, Polosan M. fMRI evidence for abnormal resting-state functional connectivity in euthymic bipolar patients. J Affect Disord. (2014) 165:182-9. doi: 10.1016/j.jad.2014.04.054

54. Dickstein DP, Gorrostieta C, Ombao H, Goldberg LD, Brazel AC, Gable CJ, et al. Fronto-temporal spontaneous resting state functional connectivity in pediatric bipolar disorder. Biol Psychiatry (2010) 68:839-46. doi: 10.1016/j.biopsych.2010.06.029

55. Ambrosi E, Arciniegas DB, Madan A, Curtis KN, Patriquin MA, Jorge RE, et al. Insula and amygdala resting-state functional connectivity differentiate bipolar from unipolar depression. Acta Psychiatr Scand. (2017) 136:129-39. doi: 10.1111 /acps.12724

56. Anticevic A, Brumbaugh MS, Winkler AM, Lombardo LE, Barrett J, Corlett PR, et al. Global prefrontal and fronto-amygdala dysconnectivity in bipolar I disorder with psychosis history. Biol Psychiatry (2013) 73:565-73. doi: 10.1016/j.biopsych.2012.07.031

57. Fernandez-Corcuera P, Salvador R, Monte GC, Sarro S, Goikolea JM, Amann $\mathrm{B}$, et al. Bipolar depressed patients show both failure to activate and failure to de-activate during performance of a working memory task. J Affect Disord. (2013) 148:170-8. doi: 10.1016/j.jad.2012.04.009

58. Townsend J, Bookheimer SY, Foland-Ross LC, Sugar CA, Altshuler LL. fMRI abnormalities in dorsolateral prefrontal cortex during a working memory task in manic, euthymic and depressed bipolar subjects. Psychiatr Res. (2010) 182:22-9. doi: 10.1016/j.pscychresns.2009.11.010

59. Brooks JO, Wang PW, Strong C, Sachs N, Hoblyn JC, Fenn R, et al. Preliminary evidence of differential relations between prefrontal cortex metabolism and sustained attention in depressed adults with bipolar disorder and healthy controls. Bipolar Disord. (2006) 8:248-54. doi: 10.1111/j.1399-5618.2006.00310.x

60. Phillips ML, Drevets WC, Rauch SL, Lane R. Neurobiology of emotion perception I: the neural basis of normal emotion perception. Biol Psychiatry (2003) 54:504-14. doi: 10.1016/S0006-3223(03)00168-9

61. Liu J, Blond BN, van Dyck LI, Spencer L, Wang F, Blumberg HP. Trait and state corticostriatal dysfunction in bipolar disorder during emotional face processing. Bipolar Disord. (2012) 14:432-41. doi: 10.1111/j.1399-5618.2012.01018.x

62. Soeiro-de-Souza MG, Salvadore G, Moreno RA, Otaduy MCG, Chaim KT, Gattaz WF, et al. Bcl-2 rs956572 Polymorphism is associated with increased anterior cingulate cortical glutamate in euthymic bipolar I disorder. Neuropsychopharmacology (2013) 38:468-75. doi: 10.1038/npp.2012.203

63. Townsend JD, Torrisi SJ, Lieberman MD, Sugar CA, Bookheimer SY, Altshuler LL. Frontal-amygdala connectivity alterations during emotion downregulation in bipolar i disorder. Biol Psychiatry (2013) 73:127-35. doi: 10.1016/j.biopsych.2012.06.030

64. Blumberg HP, Donegan NH, Sanislow CA, Collins S, Lacadie C, Skudlarski $P$, et al. Preliminary evidence for medication effects on functional abnormalities in the amygdala and anterior cingulate in bipolar disorder. Psychopharmacology (2005) 183:308-13. doi: 10.1007/s00213-0050156-7

65. Greicius MD, Menon V. Default-mode activity during a passive sensory task: uncoupled from deactivation but impacting activation. J Cogn Neurosci. (2004) 16:1484-92. doi: 10.1162/0898929042568532
66. Ongur D, Price JL. The organization of networks within the orbital and medial prefrontal cortex of rats, monkeys and humans. Cereb Cortex (2000) 10:206-19. doi: 10.1093/cercor/10.3.206

67. Phillips M, Ladouceur C, Drevets W. A neural model of voluntary and automatic emotion regulation: implications for understanding the pathophysiology and neurodevelopment of bipolar disorder. Mol Psychiatry (2008) 13:833-57. doi: 10.1038/mp.2008.65

68. Narita K, Suda M, Takei Y, Aoyama Y, Majima T, Kameyama M, et al. Volume reduction of ventromedial prefrontal cortex in bipolar II patients with rapid cycling: a voxel-based morphometric study. Prog Neuropsychopharmacol Biol Psychiatry (2011) 35:439-45. doi: 10.1016/j.pnpbp.2010.11.030

69. Wise T, Radua J, Via E, Cardoner N, Abe O, Adams TM, et al. Common and distinct patterns of grey-matter volume alteration in major depression and bipolar disorder: evidence from voxel-based meta-analysis. Mol Psychiatry (2017) 22:1455-63. doi: 10.1038/mp.2016.72

70. Wang Y, Zhong SM, Jia YB, Sun Y, Wang B, Liu T, et al. Disrupted restingstate functional connectivity in nonmedicated bipolar disorder. Radiology (2016) 280:529-36. doi: 10.1148/radiol.2016151641

71. Wang Y, Zhong S, Jia Y, Zhou Z, Zhou Q, Huang L. Reduced interhemispheric resting-state functional connectivity in unmedicated bipolar II disorder. Acta Psychiatr Scand. (2015) 132:400-7. doi: 10.1111 /acps.12429

72. Ongur D, Lundy M, Greenhouse I, Shinn AK, Menon V, Cohen BM, et al. Default mode network abnormalities in bipolar disorder and schizophrenia. Psychiatry Res. (2010) 183:59-68. doi: 10.1016/j.pscychresns.2010.04.008

73. Zhu XL, Wang X, Xiao J, Liao J, Zhong MT, Wang W, et al. Evidence of a dissociation pattern in resting-state default mode network connectivity in first-episode, treatment-naive major depression patients. Biol Psychiatry (2012) 71:611-7. doi: 10.1016/j.biopsych.2011.10.035

74. Cooney RE, Joormann J, Eugene F, Dennis EL, Gotlib IH. Neural correlates of rumination in depression. Cogn Affect Behav Neurosci. (2010) 10:470-8. doi: 10.3758/CABN.10.4.470

75. Farb NAS, Anderson AK, Bloch RT, Segal ZV. Mood-linked responses in medial prefrontal cortex predict relapse in patients with recurrent unipolar depression. Biol. Psychiatry (2011) 70:366-72. doi: 10.1016/j.biopsych.2011.03.009

76. Bartova L, Meyer BM, Diers K, Rabl U, Scharinger C, Popovic A, et al. Reduced default mode network suppression during a working memory task in remitted major depression. J Psychiatr Res. (2015) 64:9-18. doi: 10.1016/j.jpsychires.2015.02.025

77. Zhang L, Vander Meer L, Opmeer EM, Marsman JC, Ruhe HG, Aleman A. Altered functional connectivity during self- and close other-reflection in patients with bipolar disorder with past psychosis and patients with schizophrenia. Neuropsychologia (2016) 93:97-105. doi: 10.1016/j.neuropsychologia.2016.09.020

78. Onitsuka T, Shenton ME, Salisbury DF, Dickey CC, Kasai K, Toner SK, et al. Middle and inferior temporal gyrus gray matter volume abnormalities in chronic schizophrenia: an MRI study. Am J Psychiatry (2004) 161:1603-11. doi: 10.1176/appi.ajp.161.9.1603

79. Elvsashagen T, Westlye LT, Boen E, Hol PK, Andreassen OA, Boye B, et al. Bipolar II disorder is associated with thinning of prefrontal and temporal cortices involved in affect regulation. Bipolar Disord. (2013) 15:855-64. doi: 10.1111/bdi.12117

80. Lv D, Lin W, Xue Z, Pu W, Yang Q, Huang X, et al. Decreased functional connectivity in the language regions in bipolar patients during depressive episodes but not remission. J Affect Disord. (2016) 197:116-24. doi: 10.1016/j.jad.2016.03.026

81. Wang Y, Zhong SM, Jia YB, Zhou ZF, Wang B, Pan JY, et al. Interhemispheric resting state functional connectivity abnormalities in unipolar depression and bipolar depression. Bipolar Disord. (2015) 17:48695. doi: 10.1111/bdi.12315

82. Brooks JO, Vizueta N, Penfold C, Townsend JD, Bookheimer SY, Altshuler LL. Prefrontal hypoactivation during working memory in bipolar II depression. Psychol Med. (2015) 45:1731-40. doi: 10.1017/S0033291714002852

83. Cerullo MA, Eliassen JC, Smith CT, Fleck DE, Nelson EB, Strawn JR, et al. Bipolar I disorder and major depressive disorder show similar 
brain activation during depression. Bipolar Disord. (2014) 16:703-12. doi: 10.1111/bdi.12225

84. Singh MK, Chang KD, Kelley RG, Cui X, Sherdell L, Howe ME, et al. Reward processing in adolescents with bipolar I disorder. J Am Acad Child Adolesc Psychiatry (2013) 52:68-83. doi: 10.1016/j.jaac.2012.10.004

85. McCarley RW, Shenton ME, O’Donnell BF, Faux SF, Kikinis R, Nestor PG, et al. Auditory P300 abnormalities and left posterior superior temporal gyrus volume reduction in schizophrenia. Arch Gene Psychiatry (1993) 50:190-7. doi: 10.1001/archpsyc.1993.01820150036003

86. Heidrich A, Strik WK. Auditory P300 topography and neuropsychological test performance: evidence for left hemispheric dysfunction in schizophrenia. Biol Psychiatry (1997) 41:327-35. doi: 10.1016/S0006-3223(96)00030-3

87. Kalb R, Raydt G, Reulbach U, Kornhuber J. Symmetry reversal in schizophrenia. Psychiatry Clin Neurosci. (2003) 57:353-60. doi: 10.1046/j.1440-1819.2003.01131.x

88. Schroder J, Wenz F, Schad LR, Baudendistel K, Knopp MV. Sensorimotor cortex and supplementary motor area changes in schizophrenia. a study with functional magnetic resonance imaging. Br J Psychiatry (1995) 167:197-201. doi: 10.1192/bjp.167.2.197

89. Yurgelun-Todd DA, Waternaux CM, Cohen BM, Gruber SA, English CD, Renshaw PF. Functional magnetic resonance imaging of schizophrenic patients and comparison subjects during word production. Am J Psychiatry (1996) 153:200-5. doi: 10.1176/ajp.153.2.200

90. Bruder GE, Stewart JW, McGrath PJ. Right brain, left brain in depressive disorders: clinical and theoretical implications of behavioral, electrophysiological and neuroimaging findings. Neurosci Biobehav Rev. (2017) 78:178-91. doi: 10.1016/j.neubiorev.2017.04.021

91. Kemp AH, Griffiths K, Felmingham KL, Shankman SA, Drinkenburg W, Arns M, et al. Disorder specificity despite comorbidity: resting EEG alpha asymmetry in major depressive disorder and post-traumatic stress disorder. Biol Psychol. (2010) 85:350-4. doi: 10.1016/j.biopsycho.2010.08.001

92. Stewart JL, Coan JA, Towers DN, Allen JJB. Resting and task-elicited prefrontal EEG alpha asymmetry in depression: support for the capability model. Psychophysiology (2014) 51:446-55. doi: 10.1111/psyp.12191

93. Fitzgerald PB, Laird AR, Maller J, Daskalakis ZJ. A meta-analytic study of changes in brain activation in depression. Hum Brain Mapp. (2008) 29:683-95. doi: 10.1002/hbm.20613
94. Harmon-Jones E, Gable PA, Peterson CK. The role of asymmetric frontal cortical activity in emotion-related phenomena: a review and update. Biol Psychol. (2010) 84:451-62. doi: 10.1016/j.biopsycho.2009.08.010

95. Black FW. Unilateral brain lesions and MMPI performance: a preliminary study. Percept Mot Skills (1975) 40:87-93. doi: 10.2466/pms.1975.40.1.87

96. Gasparrini WG, Satz P, Heilman K, Coolidge FL. Hemispheric asymmetries of affective processing as determined by the minnesota multiphasic personality inventory. J Neurol Neurosurg Psychiatry (1978) 41:470-3. doi: 10.1136/innp.41.5.470

97. Robinson RG, Price TR. Post-stroke depressive disorders: a follow-up study of 103 patients. Stroke (1982) 13:635-41. doi: 10.1161/01.STR.13.5.635

98. Sackeim HA, Greenberg MS, Weiman AL, Gur RC, Hungerbuhler JP, Geschwind N. Hemispheric asymmetry in the expression of positive and negative emotions. Neurologic evidence. Arch Neurol. (1982) 39:210-8. doi: 10.1001/archneur.1982.00510160016003

99. Garcia-Toro M, Montes JM, Talavera JA. Functional cerebral asymmetry in affective disorders: new facts contributed by transcranial magnetic stimulation. J Affect Disord. (2001) 66:103-9. doi: 10.1016/S0165-0327(00)00276-7

100. Koek RJ, Yerevanian BI, Tachiki KH, Smith JC, Alcock J, Kopelowicz A. Hemispheric asymmetry in depression and mania. a longitudinal QEEG study in bipolar disorder. J Affect Disord. (1999) 53:109-22. doi: 10.1016/S0165-0327(98)00171-2

101. Chen X, Lu B, Yan CG. Reproducibility of R-fMRI metrics on the impact of different strategies for multiple comparison correction and sample sizes. Hum Brain Mapp. (2018) 39:300-18. doi: 10.1002/hbm.23843

Conflict of Interest Statement: The authors declare that the research was conducted in the absence of any commercial or financial relationships that could be construed as a potential conflict of interest.

Copyright (๐) 2018 Luo, Chen, Jia, Gong, Qiu, Zhong, Zhao, Chen, Lai, Qi, Huang and Wang. This is an open-access article distributed under the terms of the Creative Commons Attribution License (CC BY). The use, distribution or reproduction in other forums is permitted, provided the original author(s) and the copyright owner(s) are credited and that the original publication in this journal is cited, in accordance with accepted academic practice. No use, distribution or reproduction is permitted which does not comply with these terms. 